\title{
GPPS-BJ-2019-0146
}

\section{ROBUST OPTIMIZATION FOR VORTEX GENERATORS IN U-BEND CHANNEL OF HIGH TEMPERATURE BLADES}

\author{
Zhendong GUO \\ ericzhendong@163.com \\ Data Science and Al Research Center, Nanyang \\ Technological University, Singapore
}

\author{
Zhi TAO \\ tz1269327815@stu.xjtu.edu.cn \\ Institute of Turbomachinery, Xi'an Jiaotong \\ University, Xi'an, China
}

\author{
Liming SONG \\ songlm@mail.xjtu.edu.cn \\ Institute of Turbomachinery, \\ X'an Jiaotong University, Xi'an, \\ China
}

\author{
Jun LI \\ junli@mail.xjtu.edu.cn \\ Institute of Turbomachinery, \\ Xi'an Jiaotong University, Xi'an, \\ China
}

\author{
Zhenping FENG \\ zpfeng@mail.xjtu.edu.cn \\ Institute of Turbomachinery, \\ Xi'an Jiaotong University, Xi'an, \\ China
}

\begin{abstract}
The uncertainties such as manufacturing errors and environmental variations are inevitably encountered in engineering design, therefore in order to find robust solutions which keep high performance over a wide range, a meanvariance multi-objective robust optimization (RO) framework was proposed. Specifically, a kriging-based uncertainty quantification (UQ) formulation was proposed, which formulates uncertainty parameters and optimization design variables in product form, thereby the optimization search and UQ are conducted simultaneously in RO. Before $\mathrm{RO}$, the sequential sampling strategies were employed to build high-accurate surrogate, which was shown effective by our five-variable engineering problem. Through aero-thermal design of vortex generators (VG) in U-bend channel of hightemperature blades, some issues such as constraints selection and knowledge mining in RO space were addressed. The RO of VG was carried out with one Gaussian-distributed and one uniformly-distributed uncertainty parameter, and three optimization design variables. One deterministic optimization (DO) process at the nominal condition and several RO process with different constraints were conducted. It was shown that the proposed RO was able to find robust solutions that have high-performance and are not sensitive to uncertainty fluctuations; which were validated by CFD. Meanwhile, the solution of DO was found helpful to exclude variance-dominated solutions which have much worse performance. But some constraints based on solution of DO would make the RO solution set to be null, therefore the RO can be an iterative process, in this regard, it is attractive to build high-accurate surrogates before RO. From another perspective, the several RO with different constraints are useful to group the solutions and get a insight into black-
\end{abstract}

box design space like VG, which is valuable to select the final solution from many Pareto solutions.

\section{INTRODUCTION}

The uncertainties are always encountered in engineering design, which can come from various sources such as manufacturing error, environmental variation (e.g. the change of operational condition that different from design condition) and etc.[1]. The designs optimized purely for nominal performance may suffer from significantly degraded performance when uncertainty propagates, e.g., Bunker [2] showed that the geometric uncertainties can lead to a temperature increase of $40 \mathrm{~K}$ at most for high-temperature blades, which should reduce the life of aero-engines by $30 \%$. D'Ammaro and Montomoli [3] investigated the effects of fluctuations of operation condition on film-cooling effectiveness of a gas turbine blade; he showed that a $20 \%$ stochastic variation of the inlet total pressure gave a variation of the adiabatic effectiveness of about $80 \%$. Therefore, extensive attentions [1, 4] have been drawn to the robust design, which seeks to obtain solutions that keep high performance over a wide range.

Both the probabilistic approaches and deterministic methods (which incorporate non-statistical indices such as gradients [5] or sensitivities [6]) are developed for robust design. Our focus is on probabilistic robust optimization (RO), then the following four issues such as (a) uncertainty quantification (UQ), (b) dealing with uncertainty and optimization variables, (c) robustness measurement and (d) surrogate accuracy management in RO needs to be considered. As for UQ, the Monte Carlo simulation (MCS) [7] is most commonly used which is straightforward. However, the MCS requires hundreds of thousands and even millions of function calls, which is a prohibitive cost for 
expensive problems if only direct simulations (e.g. CFD) were used for the performance evaluation. Therefore, some efforts were drawn to use surrogates (e.g. hierarchal kriging [8]) instead of direct simulations for MCS or directly build surrogates [9, 10] of mean and variance. Alternatively, people resort to develop formulations of mean and the variance directly by polynomial chaos expansion $[11,12]$ and kriging [13-15] as well.

Concerning the problem as how to deal with optimization design variables and uncertainty parameters, they are often merged as uncertainty variables in most references [11-18], and doubled loop optimization strategies are frequently adopted, i.e., the variables are searched in a large space to obtain better nominal performance in an outer loop. Then, by varying corresponding variables of optimized solution in its neighborhood of small range, UQ was conducted in an inner loop. Most of the UQ methods [8, 9, 11, 13-15] shown in the previous paragraph are designed for the uncertainty variables, which doesn't distinguish between optimization variables and uncertainty parameters.

On the other hand, there exists situation when optimization design variables and uncertainty parameters cannot be merged, e.g. for RO of drag coefficient of wing RAE 2822 in [12], the shape parameters of the airfoil was to be optimized, while the uncertainties that come from fluctuation of operation conditions such Mach number and attack angle are considered for design robustness. Our proposed UQ formulation is aimed for such situations, instead of taking uncertainty fluctuations as latent parameters in stochastic kriging [10]or using polynomials chaos[12], we use ordinary kriging to formulate optimization design variables and uncertainty parameters in product form, which help to conduct the optimization search and UQ simultaneously in RO.

When it comes to the robustness measurement, it can be divided into two issues. The first issue is the RO objectives and corresponding optimization strategies. The mean and variance are most frequently used for probabilistic optimization framework; both the strategies of multiobjective optimization [9, 10, 16-18] and weighted forms of mean and variance in single-objective optimization [11-15] were reported in the references. By comparing to some single-objective RO, Ryan et al. [18] stated that the multiobjective strategy was able to cover the solutions of singleobjective RO while capturing tradeoffs within the design space.

The second issue related to robustness measurement is the selection of constraints, which is rarely discussed in publications. As will be shown in the design of vortex generator (VG) in U-bend channel, when using meanvariance multi-objective strategy to conduct RO, some variance dominated "inferior" solutions can be obtained: these solutions are significantly less sensitive to the uncertainty fluctuations, but their design performance are much worse. To exclude these "inferior" solutions, constraints should be imposed for RO. However, most engineering problems we encountered are black box [19], i.e., the relations between variables and objectives cannot be explicitly expressed; we do not have much prior knowledge on what kind of constraints should be effective. Then the following problems would occur: on one hand, the constraints set by original design may not be able to filter out the "inferior" solutions. Actually, we can add uncertainty information from solution of deterministic optimization (DO) to exclude RO solutions that not competitive to the solution of DO. However, on the other hand, the constraints imposed by solution of DO may be too strong to make the set of RO solutions to be null. Then, the RO has to iterate several times when null solution set was encountered. Further, as will be addressed in our engineering example, for design space of black-box, doing the RO several times with different constraints is beneficial, the solutions can be clustered by comparing the RO models (with different constraints), which is of great value to get insights into the design space $[19,21]$ and help to select the final solution from many Pareto solutions.

Finally, regarding to surrogate accuracy management in RO, Arendt et al. [13] and Sun et al.[15] propose to adaptively infill samples and update surrogate accuracy during the RO process, which was shown effective for oneshort RO. Alternatively, for expensive black-box problems, it is also attractive to build high-accurate surrogate before RO, as the RO would be conducted several times to gain more knowledge of black-box design space. With high-accurate surrogate before RO, we can efficiently conduct RO with different constraints without worrying large increase on computational cost. Further, recalling the strategies for efficient global optimization (EGO), the adaptive sequential sampling by using expected improvement (EI) function [22] and etc. is effective to tradeoffs between improving surrogate accuracy and searching optimal solutions. By infilling multiple points at each iteration as proposed by Viana et al. [23] and Zhan et al.[24], the global search performance of classic EGO algorithm [22] becomes significantly better, and one reason behind such great improvement in search performance is that, the corresponding surrogate accuracy is greatly improved. In this regard, we can use these multiple adaptive sampling methods purely to build high-accurate surrogate before RO, which will be discussed in this paper.

In addition to addressing the issues of $\mathrm{RO}$, the engineering purpose of $\mathrm{RO}$ of $\mathrm{VG}$ is to develop novel structures in U-bend internal cooling channel, in order to enhance heat transfer there and thus improve the cooling performance of high-temperature blades. The following sections are organized as follows: The kriging-based UQ and flowchart of RO were introduced in Section II, the adaptive infill sampling strategy to build high-accurate surrogate and multi-objective evolution algorithm were illustrated. Then, the parameterization and CFD model of VG were introduced in Section III. Next, upon building high-accurate surrogate for the space of both uncertainty parameters and optimization variations, DO of VG was conducted at nominal condition in Section IV. And RO of VG and was carried out in Section V, some RO issues were addressed. Finally, conclusions were made in Section VI. 


\section{UNCERTAINTY QUANTIFICATION AND ROBUST OPTIMIZATION PROCESS}

\section{UQ for Input Uncertainties of Arbitrary Continuous Probability Distributions}

The kriging is one of the most popular surrogates, whose prediction $Y$ at unknown site $\mathbf{w}$ is built as a trend function $f(\mathbf{w})$ plus a normal random process $Z(\mathbf{w})$ as:

$$
Y(\mathbf{w})=f(\mathbf{w})+Z(\mathbf{w})
$$

where, $f(\mathbf{w})$ is often taken as a constant as $\beta_{0}$, and $Z(\mathbf{w})$ describes the local features of $Y$ around the $n$ sample points $X=\left\{\mathbf{x}^{(1)}, \cdots \mathbf{x}^{(n)}\right\}$, which has zero mean and a covariance function, which usually of the form:

$$
\operatorname{cov}\left[Z(\mathbf{w}), Z\left(\mathbf{x}^{(i)}\right)\right]=\sigma^{2} \exp \left(-\sum_{h=1}^{k} \theta_{h}\left\|w_{h}-x_{h}^{(i)}\right\|^{2}\right)
$$

where, $k$ denotes the number of variables, $\sigma^{2}$ is the process variance of samples, and $\theta_{h}$ represents the curve bumpiness hyper-parameter along dimension $h$ [21,22].The kriging prediction at unknown site $\mathbf{w}$ is formulated as :

$$
\begin{aligned}
& y(\mathbf{o}, \mathbf{u})=\beta_{0}+\sum_{i=1}^{n}\left[R_{i}^{-1}\left(\mathbf{y}-\mathbf{l} \beta_{0}\right)\right] \underbrace{\exp \left[-\sum_{h=1}^{m} \theta_{h}\left\|o_{h}-x_{h}^{(i)}\right\|\right]}_{\text {Optimization design variables }} \underbrace{\exp \left[-\sum_{g=1}^{l} \theta_{g}\left\|u_{g}-x_{g}^{(i)}\right\|\right]}_{\text {Uncertainty parameters }} \\
& =\beta_{0}+\sum_{i=1}^{n} A^{(i)} \underbrace{\exp \left[-\sum_{g=1}^{l} \theta_{g}\left\|u_{g}-x_{g}^{(i)}\right\|\right]}_{\text {Uncertainty parameters }} \quad \text { where, } A^{(i)}=\left[R_{i}^{-1}\left(\mathbf{y}-\mathbf{l} \beta_{0}\right)\right] \underbrace{\exp \left[-\sum_{h=1}^{m} \theta_{h}\left\|o_{h}-x_{h}^{(i)}\right\|\right]}_{\text {Optimization Parameters }} \\
& \mu(\tilde{\mathbf{o}}, \mathbf{u})=\iint[\beta_{0}+\sum_{i=1}^{n} A^{(i)} \underbrace{\exp \left[-\sum_{g=1}^{l} \theta_{g}\left\|u_{g}-x_{g}^{(i)}\right\|\right]}_{\text {Uncertainty parameters }}] P(\mathbf{u}) d \mathbf{u} \\
& =\beta_{0}+\sum_{i=1}^{n} A^{(i)} \iint \exp \left[-\sum_{g=1}^{l} B_{g}^{(i)}\right] P(\mathbf{u}) d \mathbf{u} \quad \text { where } B_{g}^{(i)}=\theta_{g}\left\|u_{g}-x_{g}^{(i)}\right\| \\
& \sigma^{2}(\tilde{\mathbf{o}}, \mathbf{u})=\iint[\beta_{0}+\sum_{i=1}^{n} A^{(i)} \underbrace{\exp \left[-\sum_{g=1}^{l} \theta_{g}\left\|u_{g}-x_{g}^{(i)}\right\|\right]}_{\text {Uncertainty parameters }}-\mu]^{2} P(\mathbf{u}) d \mathbf{u} \\
& =-\left(\beta_{0}-\mu\right)^{2}+\sum_{i=1}^{n} A^{(i) 2} \iint \exp \left[-\sum_{g=1}^{l} C_{g}^{(i)}\right] P(\mathbf{u}) d \mathbf{u}+2 \sum_{\substack{i=1 \\
j \neq i}}^{n} A^{(i)} A^{(j)} \iint \exp \left[-\sum_{g=1}^{l} D_{g}^{(i, j)}\right] P(\mathbf{u}) d \mathbf{u} \\
& \text { where, } C_{g}^{(i)}=2 \theta_{g}\left\|u_{g}-x_{g}^{(i)}\right\|, D_{g}^{(i, j)}=\theta_{g}\left(\left\|u_{g}-x_{g}^{(i)}\right\|+\left\|u_{g}-x_{g}^{(j)}\right\|\right)
\end{aligned}
$$

$$
\begin{aligned}
& \begin{aligned}
\mathrm{y}(\mathbf{w})= & \beta_{0}+\mathrm{Z}(\mathbf{w})=\beta_{0}+\mathbf{r}^{t}(\mathbf{w}) R^{-1}\left(\mathbf{y}-\mathbf{l} \beta_{0}\right) \\
= & \beta_{0}+\sum_{i=1}^{n} R_{i}^{-1}\left(\mathbf{y}-\mathbf{l} \beta_{0}\right) \exp \left[-\sum_{h=1}^{k} \theta_{h}\left\|w_{h}-x_{h}^{(i)}\right\|\right]
\end{aligned} \\
& s^{2}(\mathbf{x})=\sigma_{z}^{2}\left[1-\mathbf{r}^{t}(\mathbf{x}) \mathrm{R}^{-1} \mathbf{r}^{t}(\mathbf{x})+\frac{\left(1-\mathbf{l}^{t} R^{-1} \mathbf{r}^{t}(\mathbf{x})\right)^{2}}{\mathbf{l}^{t} R^{-1} \mathbf{l}}\right]
\end{aligned}
$$

where, $\mathbf{y}$ denotes the function vector of given samples $X, \mathbf{l}$ is vector of ones, which is of the same size as $\mathbf{y}$. More details kriging and its implementations can be found in [21][21].

When both (non-probabilistic) design variables (denoted $\boldsymbol{o}=\left[\begin{array}{llll}o_{1}, & o_{2}, & \cdots & o_{\mathrm{m}}\end{array}\right]$ and probabilistic uncertainty ers (denoted by $\mathbf{u}=\left[u_{1}, u_{2}, \cdots u_{1}\right]$ ) are considered to e mean performance (denoted by $\mu$ ) and corresponding (denoted by $\sigma^{2}$ ) can be calculated by Eqs.(6) and (7), when the probability distributions of uncertainty parameters is given as $P(\mathbf{u})$.

Table 1 Calculation of Integrals for $\mu$ and $\sigma^{2}$ with different probability distribution functions

\begin{tabular}{ccc} 
& $P(\mathbf{u})=\prod_{i=1}^{l} P\left(u_{i}\right)$ & $P(\mathbf{u})=P\left(u_{1}\right) \times \cdots P\left(u_{g}, u_{k}\right) \times \cdots$ \\
\hline Integral of $B_{g}^{(i)}$ & $\prod_{g=1}^{l}\left[\int \exp \left(-B_{g}\right) P\left(u_{g}\right) d u_{g}\right]$ & {$\left[\int \exp \left(-B_{1}\right) P\left(u_{1}\right) d u_{1}\right] \times \cdots\left[\int \exp \left(-\left(B_{g}+B_{k}\right)\right) P\left(u_{g}, u_{k}\right) d u_{g} d u_{k}\right] \times \cdots$} \\
Integral of $C_{g}^{(i)}$ & $\prod_{g=1}^{l}\left[\int \exp \left(-C_{g}\right) P\left(u_{g}\right) d u_{g}\right]$ & {$\left[\int \exp \left(-C_{1}\right) P\left(u_{1}\right) d u_{1}\right] \times \cdots\left[\int \exp \left(-\left(C_{g}+C_{k}\right)\right) P\left(u_{g}, u_{k}\right) d u_{g} d u_{k}\right] \times \cdots$} \\
Integral of $D_{g}^{(i, j)}$ & $\prod_{g=1}^{l}\left[\int \exp \left(-D_{g}\right) P\left(u_{g}\right) d u_{g}\right]$ & {$\left[\int \exp \left(-D_{1}\right) P\left(u_{1}\right) d u_{1}\right] \times \cdots\left[\int \exp \left(-\left(D_{g}+D_{k}\right)\right) P\left(u_{g}, u_{k}\right) d u_{g} d u_{k}\right] \times \cdots$}
\end{tabular}

Further, Table 1 lists the details for input uncertainties of various probability distributions. When the uncertainty parameters are independently distributed (as often assumed in UQ problems [25]) the high-dimensional integral in Eqs. (6) and (7) can be simplified to as product of the singlevariable integral for each uncertainty parameter. Specific 
probability distribution function (abbreviated as PDF) can be assigned to each uncertainty parameter.

In addition, concerning the joint effects of uncertainty parameters, joint PDFs would be assigned, as seen in the second column; then it is obvious that the dimension of integral calculation that needs to be conducted depends on the dimension of the joint PDFs for uncertainty parameters. On the other hand, according to the research of Schonlau and Welch [26], the joint effects of three or more parameters are often weak for most well-defined systems, which is also observed in the applications of analysis of variance[21,25]. In other words, usually only the two-variable joint effects should be considered in well-defined applications, and hence the dimension of integral calculation can research to 2 at most. In other words, the high-dimensional integral can be avoided by using Eqs. (6) and (7). The techniques such as Gauss-Legendre quadrature and Simpson's rule were employed to calculate these one- and two-dimensional integrals. Comparing to MCS, the mean and variance calculated by Eqs. (6) and (7) should be much more efficient.

\section{Multi-Objective Robust Optimization Process}

Figure 1 shows the flowchart of the robust optimization process. It consists of four stages. First of all, a surrogate of high-accuracy will be built, by combing intelligent adaptive sampling and space-filling sampling strategies, the details will be illustrated through 1D toy problems. Secondly, by fixing uncertainty parameters, deterministic global optimization will be conducted at the nominal condition, and then UQ will be conducted for the solution of DO, which intends to exclude "insensitive" RO solutions which actually have much worse performance. The importance of statistical UQ information of DO solutions for RO will be discussed in detail in optimization design for VG in Section V.

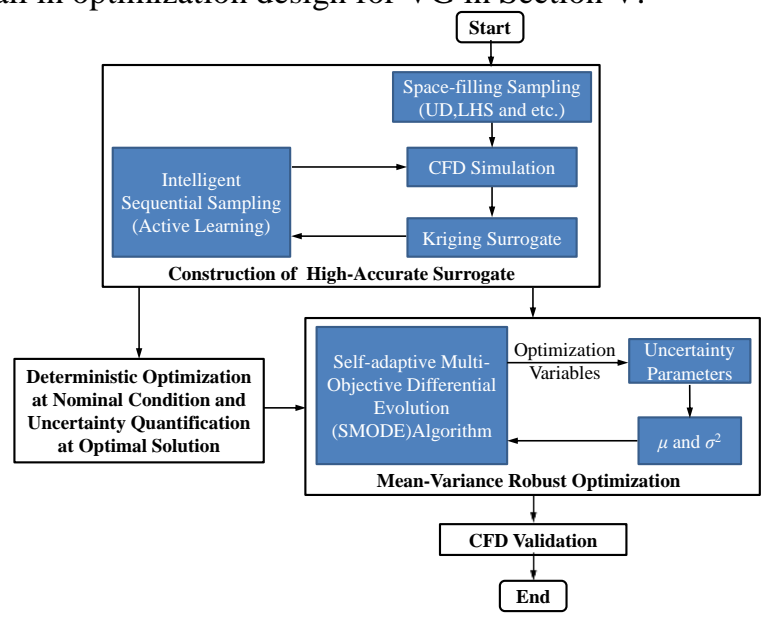

Fig.1 Framework of robust optimization process for vortex generators in U-bend channel

Thirdly, the mean-variance multi-objective RO will be conducted after DO. In this work, the uncertainty factors such as fluctuations of operation condition and manufacturing errors are considered, which are separable from design optimization variables. Therefore, as shown in Fig.2, the optimization and uncertainty quantification are conducted simultaneously in a single loop, by using Eqs. (6) and (7). This is different from conventional RO process, which usually has inter- and outer-loop for optimization and uncertainty quantification, successively. Finally, to demonstrate the effectiveness of RO, the better performance of optimal Pareto solutions w.r.t. reference design and optimal solution of DO as well will be validated using CFD simulations.

To build accurate kriging for UQ and optimization process, two strategies are considered. First, the vertices of hyper-rectangle space are included, which is effective to reduce the probabilities of extrapolation, as shown in Fig.3. However, it should be noted that, with the increase of dimensions (denoted by $n$ ), the number of vertices will be dramatically increased by $2^{n}$, therefore the strategy to include vertices is most useful for the design space with the number of variables less than 10 .

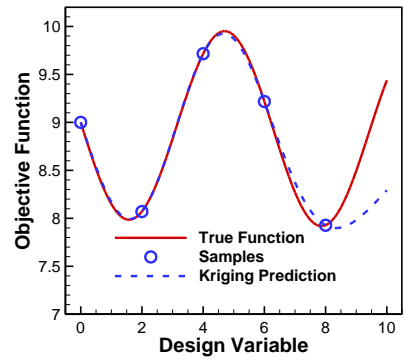

(a) Not all vertices included

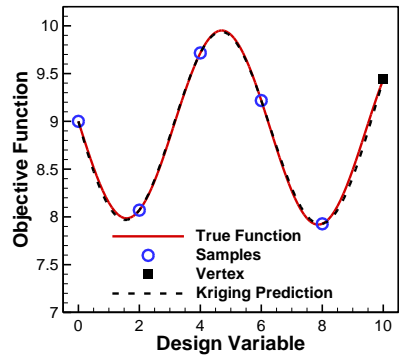

(b) Including vertices
Fig.2 Including vertices to reduce the probability of extrapolation

Second, in order to adaptively improve the surrogate accuracy in local areas, several intelligent infill criterions such as generalized expected improvement (GEI), mean squared error of surrogate $\left(s^{2}\right)$ and best solution of current surrogate $\left(\hat{f}_{\min }\right)$ are combined. Equation (8) shows the expression of expected improvement (EI), which calculates the possibilities of function value at unknown sites to be better than current optimal solution. By adding a global factor $g$, a generalized form of EI (GEI) function was proposed by Schonlau [26], as shown in Eq. (9). Then, a combination of such infill criterions is proposed in paper for multi-points sampling over space, as seen in Eq. (10).

$$
\begin{aligned}
E I(\mathbf{x}) & =\int_{-\infty}^{f_{\min }}\left(Y(\mathbf{x})-f_{\min }\right) d Y \\
& =\left(f_{\min }-\hat{y}(x)\right) \Phi\left(\frac{f_{\min }-\hat{y}}{s}\right)+s \phi\left(\frac{f_{\min }-\hat{y}}{s}\right) \\
G E I(\mathbf{x}) & = \begin{cases}P\left(\frac{y-\hat{y}}{s}<u\right) & g=0 \\
s^{g} \sum_{k=0}^{g}(-1)^{k}\left(\begin{array}{l}
g \\
k
\end{array}\right) u^{g-k} T_{g} & 1 \leq g \leq 10\end{cases}
\end{aligned}
$$

Infill points $=\left\{\max [G E I(\mathbf{x})], \max \left[s^{2}(\mathbf{x})\right], \max \left[-\hat{f}_{\min }(\mathbf{x})\right]\right\}$

To be noted, the EI $(g=1)$ and GEI functions are originally used for surrogate-based efficient global optimization. However, the EI and GEI functions are formulated by taking a balance between surrogate accuracy uncertainties and current optimal solution; they are effective in improving the surrogate accuracy at unknown areas, as 
shown in improved version of EGO [23, 24]. Ponweiser et al. [27] point out that, the GEI with larger $g$ will put much emphasize on unknown regions with few samples, so does $s^{2}$. On the contrary, the EI and $\hat{f}_{\text {min }}$ are likely to infill samples near the surrogate optimum and thus improve the surrogate accuracy there. Thereby, it can be expected that a highaccurate surrogate over space can be obtained simultaneously while finding optimal solutions. Such favorable features are employed for UQ and RO process here, aiming to build highaccuracy kriging.

Figure 3 shows the effectiveness of sequential sampling. In Fig.3 (a), the dash-dotted lines are curves of infill criterions in (10). For easy observation, two typical of them are bolded to show their ability to take care of both unknown areas as (a) regions with relatively fewer samples (and thus in bad accuracy) and (b) regions near the surrogate optimum. The overlapped infill points are filtered out by setting a distance threshold. Then, by adding the adaptive infill points, the accuracy of updated kriging is observed significantly improved in Fig.3 (b).

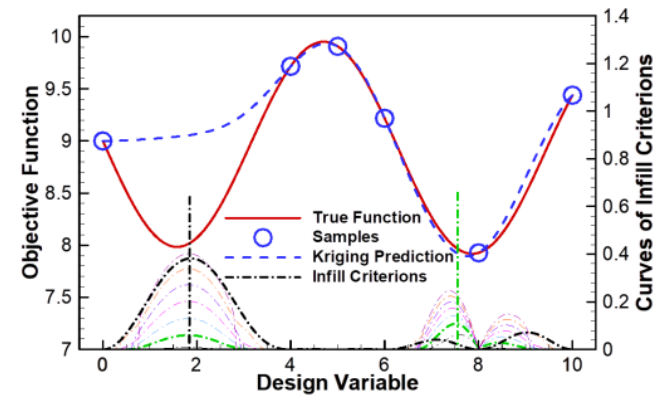

(a) Initial kriging and potential infill samples

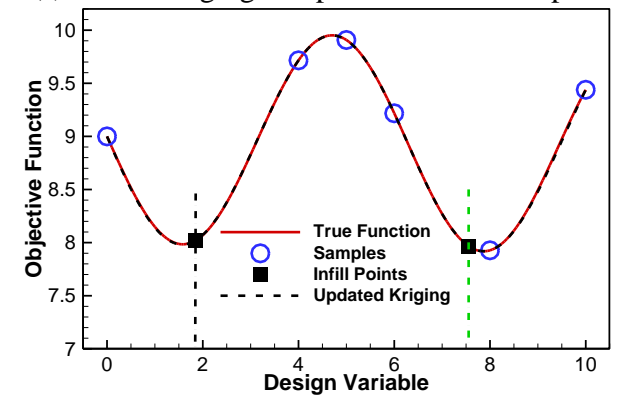

(b) Updated kriging

Fig.3 Intelligent sequential sampling to improve surrogate accuracy over space

For the multi-objective robust optimization, a selfadaptive multi-objective evolution algorithm named as SMODE, was employed in this work. More details can be found in Ref. [28].

\section{PARAMETERIZATION AND CFD SIMULATION OF VG IN U-BEND CHANNEL}

\section{Geometric Model and Parameterization of VG for Optimization}

Figure 4 shows the computational model of Delta-Shaped VG in U-bend channel, and Fig. 5 and Table 2 define the geometric specifications by referring to [29]. The crosssectional aspect ratio of the channel $(\mathrm{W} / \mathrm{H})$ is $1: 2$; thereby the corresponding hydraulic diameter of the cross section is $16.93 \mathrm{~mm}$. In order to improve the thermal performance, 10 delta-shaped VG are equipped in both in- and out-flow path of the U-bend channel. The distance from the channel inlet to the leading edge of the first VG (denoted by $d$ ) is $6.35 \mathrm{~mm}$, and the distance between each VG (denoted by p) is 14.00 $\mathrm{mm}$, these distances will be fixed during the optimization.

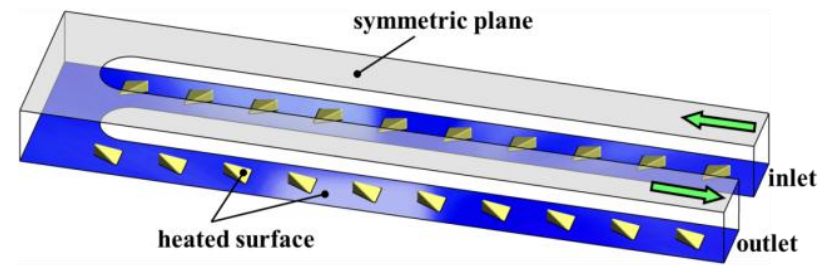

Fig.4 Computational model of the U-bend Channel equipped with delta-shaped vortex generators
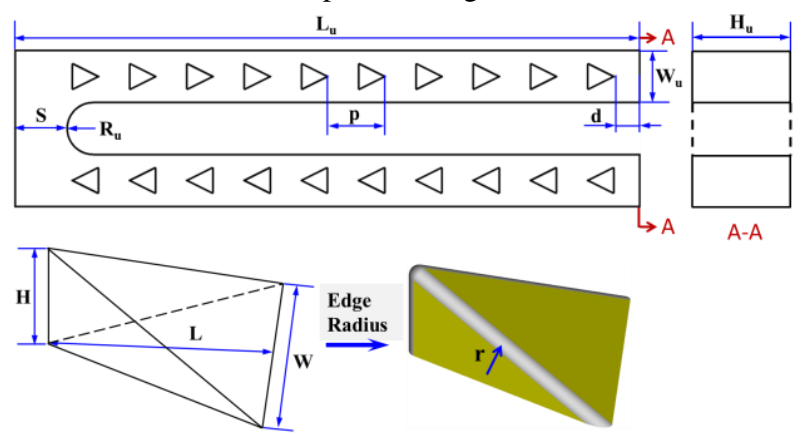

Fig. 5 Geometry of U-bend channel equipped with delta-shaped vortex generators

Table 2 Geometric specifications of the U-bend channel

\begin{tabular}{lccccc}
$\begin{array}{l}\text { Geometric } \\
\text { Parameters }\end{array}$ & $L_{u}$ & $W_{u}$ & $H_{u}$ & $R_{u}$ & $S$ \\
\hline Values/mm & 152.4 & 12.7 & 25.4 & 6.35 & 12.7 \\
\hline
\end{tabular}

For the optimization, the height $(H)$, length $(L)$ and width $(W)$ of the VG will be adjusted as optimization variables to improve the aero-thermal performance of U-bend channel, while the size of $10 \mathrm{VGs}$ will keep in the same size. The variable range of $H, L$ and $W$ in optimization will be given in Section IV. For robust optimization, the dimensional tolerance of edge fillet (abbreviated as $r$ ) is considered, and the fillet radius is set to be $0.3 \mathrm{~mm}$ at nominal condition according to [30]. In addition, the Reynolds number (abbreviated as $R \mathrm{e}$ ) is taken as another uncertainty parameter, the fluctuation ranges of $r$ and $R$ e are given in Section $V$, where the robust optimizations are carried out.

\section{CFD Method and Numerical Validation}

The commercial software, ANSYS CFX 14.5, is used for the aero-thermal performance evaluation of U-bend channel. The Reynolds-Averaged Navier-Stokes (RANS) solver is employed. Figure 4 shows the computational domain of the U-bend channel. The boundary conditions are set in accordance with the experimental test [29]. Specifically, a uniform velocity profile is imposed at the channel inlet. The inlet velocity is calculated according to the inlet Reynolds number, and the inlet temperature is fixed at $298.15 \mathrm{~K}$, while the inlet turbulence intensity is set as $5 \%$. The non-slip wall with a constant temperature of $338.15 \mathrm{~K}$ is given at the 
channel heated wall surface. Considering the symmetry of the U-shaped channel along the span, a symmetric condition is imposed at the channel mid-span (see Fig. 4). An averaged static pressure is imposed at outlet. To ensure computational convergence, the residuals for continuity, energy, and the area-averaged wall temperature are set as less than $10^{-5}, 10^{-5}$, and $10^{-3}$, respectively. Concerning for the mesh grids, the $\mathrm{C}$ type grids are used for the regions around the channel turn and the Y-type grids are generated for the surface of deltashaped VG. The distance of the first cell to the wall is set to be $0.002 \mathrm{~mm}$ correspondingly, the dimensionless distance of the first cell to the wall [31], which is often denoted by $y^{+}$, is less than 1.

The Mesh sensitivity analysis is conducted at the nominal Reynolds number of 25000 with four different mesh sizes as 2.4 million, 3.1 million, 3.9 million and 4.6 million, and the performance is evaluated with the most popular turbulence model, SST $k-\omega$. As shown in the last column of Table 3, the deviation of two critical performance indicators as the area-averaged Nusselt number augmentation ratio $\left(\mathrm{Nu} / \mathrm{Nu} u_{0}\right)$ and the friction ratio $\left(f / f_{0}\right)$ are less than $0.5 \%$. It indicates that the grids size of 4.6 million is sufficient to get rid of the influence of mesh dependence for the evaluation. Therefore, the 4.6 million-mesh is adopted for the optimization process, and a grid template is set up to ensure similar mesh topology and mesh quality in design process.

Table 3 Mesh sensitivity analysis

\begin{tabular}{ccccc}
\hline Mesh /million & 2.4 & 3.1 & 3.9 & 4.6 \\
\hline$N u / N u_{0}$ & 1.842 & 1.885 & 1.907 & 1.916 \\
Difference/\% & 3.86 & 1.62 & 0.46 & Baseline \\
$f / f_{0}$ & 3.21 & 3.165 & 3.136 & 3.131 \\
Difference/\% & 2.53 & 1.08 & 0.12 & Baseline \\
\hline
\end{tabular}

Further, the turbulence model is validated against Han's test data [29]. Three different turbulence models as the standard $k-\varepsilon$ model, the RNG $k-\varepsilon$ model, and the SST $k-\omega$ model are chosen, which were conducted with 4.6million-mesh.

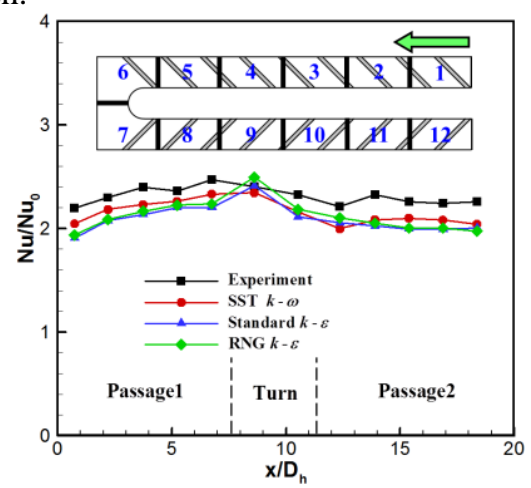

Fig. 6 Numerical Validation of Turbulence models against experimental test

Figure 6 compares the regional area-averaged Nusselt number augmentation ratio on the ribbed wall with the data from [29]. Obviously, the trend and values predicted by SST $k-\omega$ model is most close to experimental data. Meanwhile, the largest discrepancy between the numerical results and test data is less than $8.9 \%$. Therefore, the accuracy of SST $k-\omega$ model is confirmed and selected for performance evaluation in optimization.

\section{SURROGATE MODELING AND DETERMINISTIC OPTIMIZATION FOR VG}

\section{Surrogate Modeling and Cross Validation}

As discussed in Section III.A, the height $(H)$, width $(W)$ and length $(L)$ of delta-shaped VG will be used as optimization variables to improve the aero-thermal performance of U-bend channel, while the dimensional tolerance of fillet radius $(r)$ and the fluctuation of Reynolds number $(R \mathrm{e})$ will be taken as uncertainty parameters, therefore there are five variables in total to build the kriging surrogate. The objective is the overall thermal performance (abbreviated as TP), which measures the heat-transfer performance of U-bend channel by trading off the aerodynamic loss.

For the five-variable space, there are 68 samples in total. Where, 29 of them are generated by using a space-filling technique, uniform design, which are shown in circles in Fig.7. Meanwhile, the $32\left(2^{5}\right)$ vertices are shown in diamonds. In addition, 6 samples (denoted by deltas) are successively generated by sequential sampling in three iterations. Note that circles are initial space-filling samples, squares are vertices and deltas are generated by intelligent sequential sampling

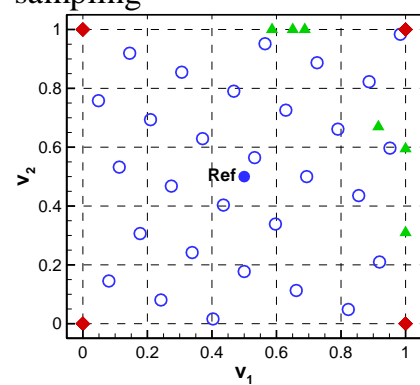

Fig.7 Projection of samples in normalized 2D space

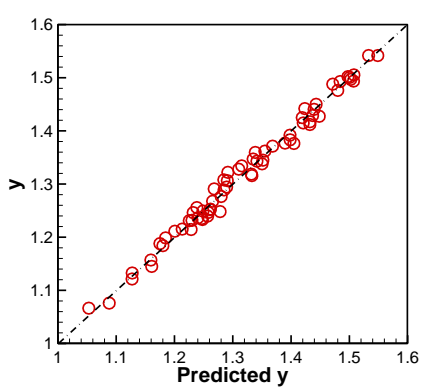

Fig. 8 Cross validation of kriging predictions
To validate the accuracy of kriging prediction, the leaveone-out cross validation was conducted as shown in Fig.8. Obviously, the scatters which consists of true and predicting values as (Predict y, y) distributed closely to the $45 \mathrm{deg}$ line, it means the thermal objective of U-bend channel, TP, can be well predicted by kriging. In addition, the cross-validation based RMSE and $R^{2}$ of the kriging are 0.01314 and 0.9871 , which confirms the high-accuracy of TP kriging. The effectiveness of intelligent adaptive sampling will be further discussed by CFD validation in Section VI. Using this kriging, the deterministic optimization and robust optimization will be carried out in following sections.

\section{Deterministic Optimization at Nominal Condition}

The kriging-based EGO algorithm is used for the deterministic optimization. The objective of deterministic optimization is conducted for the maximization of overall thermal performance, which is defined as below:

$$
T P=\left(N u / N u_{0}\right) /\left(f / f_{0}\right)^{\frac{1}{3}}
$$


The range of optimization variables are shown in Table 4, while the uncertainty parameters, $R$ e and $r$, are fixed at the nominal condition, as shown in the last two columns.

Table 4 The range of optimization variables at nominal condition

\begin{tabular}{cccccc}
\hline Variables & $x_{1}$ & $x_{2}$ & $x_{3}$ & $u_{1}$ & $u_{2}$ \\
\hline $\begin{array}{c}\text { Geometric } \\
\text { parameters } \\
\begin{array}{c}\text { Reference } \\
\text { design }\end{array}\end{array}$ & $L / \mathrm{mm}$ & $W / \mathrm{mm}$ & $H / \mathrm{mm}$ & $R \mathrm{e}$ & $r / \mathrm{mm}$ \\
$\begin{array}{c}\text { variable range } \\
\text { vabla }\end{array}$ & $4 \sim 9$ & $4 \sim 9$ & $2 \sim 5$ & -- & -- \\
\hline
\end{tabular}

After optimization and validation by CFD, the TP of the (deterministic) optimal solution at nominal condition is 1.4969 , which is $10.1 \%$ higher than that of the reference design (TP of reference design is 1.3595).

Figure 9 compares the $\mathrm{Nu} / \mathrm{Nu}$ ( see Apendix D) contours on the heated surface (see Fig.6) of U-bend channel at nominal condition. Obviously, the heat transfer performance of optimal solution was greatly enhanced, as the $\mathrm{Nu} / \mathrm{Nu} u_{0}$ of the optimal solution is much larger in outflow path of U-bend channel. The covering range of high $\mathrm{Nu} / \mathrm{Nu}$ is also increased in $y$ direction at both in- and outflowpath, as seen in Fig.9.

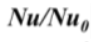

$\begin{array}{lllllllllll}0.0 & 0.5 & 1.0 & 1.5 & 2.0 & 2.5 & 3.0 & 3.5 & 4.0 & 4.5 & 5.0\end{array}$

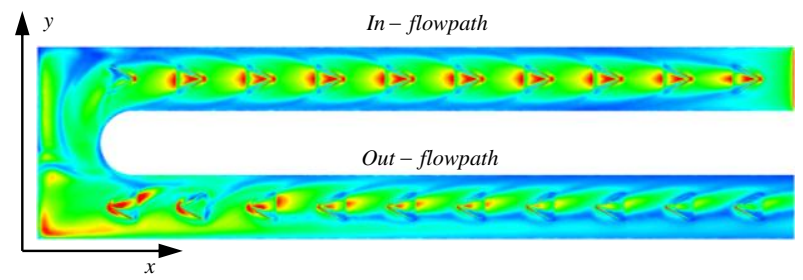

(a) Reference design

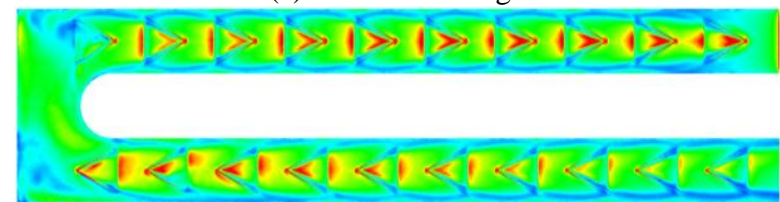

(b) Deterministic optimal solution

Fig. 9 Comparison of $N u / N u_{0}$ contours on heated surface at nominal condition

Further, the thermal performance and the flow resistance in U-bend channel are separately analyzed in Fig.10. Obviously, there should have some tradeoffs on the way to improve the overall thermal performance (TP), the $\mathrm{Nu}$ of optimal solution was increased (see Fig.10 (a), but it also need more mechanical work to against the increased flow resistance (see Fig.10 (b)). When compared to the classic heat-transfer-enhanced structures such as the angled ribs in Han's work[29], the heat transfer performance of our optimal solution is better while the flow resistance is also smaller at different $R \mathrm{e}$ conditions. It indicates that the VGs we proposed in U-bend channel should be a competitive internal cooling structure to resolve the cooling problem of hightemperature blades.

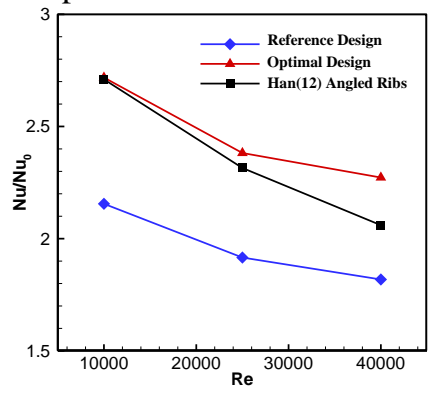

(a) Average $\mathrm{Nu} / \mathrm{Nu}$

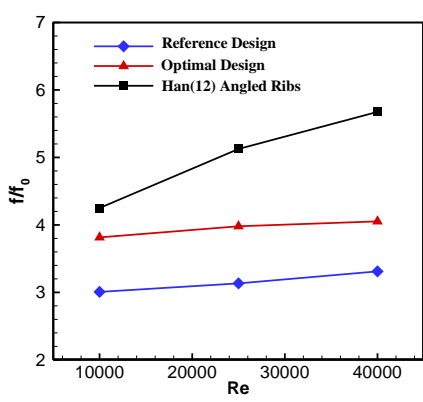

(b) Average $f / f_{0}$
Fig.10 Thermal and aerodynamic performance comparison

\section{ROBUST OPTIMIZATION FOR VG}

As already noted in previous sections, the dimensional tolerance of fillet radius ( $r$ ) on VG (see Fig.5) and the operational fluctuation of $R \mathrm{e}$ are considered as uncertainty parameters, the objective of $\mathrm{RO}$ is to maximize the mean of $\mathrm{TP}$, and to minimize the corresponding variance when uncertainties propagates. The uncertainty information of the solution obtained by DO (denoted as DO_Opt) is taken as a reference to inspect the advantage of RO w.r.t DO. Several $\mathrm{RO}$ process were conducted to gain an insight into the RO design space.

Table 5 shows the range of optimization variables and uncertainty parameters. The optimization variables keep the same as in DO process (see Table 5). The fluctuation range of $R \mathrm{e}$ is given by referring to [3], while the range of $r$ is set according to [2]. Both the Re and $r$ varies around $\pm 20 \%$ of its nominal value, and they follow uniform (abbreviated as $\mathrm{U}$ in Table 5) and normal distribution (abbreviated as $N$ ), respectively.

Table 5 Range of optimization variables and uncertainty parameters in robust optimization process

\begin{tabular}{|c|c|c|c|c|c|}
\hline \multirow{2}{*}{ Variables } & \multicolumn{3}{|c|}{ Optimization variables } & \multicolumn{2}{c|}{ Uncertainty parameters } \\
\cline { 2 - 6 } & $x_{1}$ & $x_{2}$ & $x_{3}$ & $u_{1}$ & $u_{2}$ \\
\hline Geometric parameters & $L / \mathrm{mm}$ & $W / \mathrm{mm}$ & $H / \mathrm{mm}$ & $r / \mathrm{mm}$ & 0.30 \\
\hline Reference design at nominal condition & 6 & 6 & 3 & 25000 & $N(0.3,0.02)$ \\
\hline Variable range & $4 \sim 9$ & $4 \sim 9$ & $2 \sim 5$ & $U[20000,30000]$ & \\
\hline
\end{tabular}

\section{Basic RO Process}

First, supposing that the DO was not conducted; we directly employ the RO algorithm to explore robust solutions of VG that can enhance the heat transfer performance of Ubend channel. In addition, the RO design space is a "blackbox" at this initial stage, we basically assume that both the mean and variance values of optimized RO solutions should be better than the reference design in Section III. The corresponding RO model is as follows:

$$
\begin{aligned}
& \max \left\{\mu,-\sigma^{2}\right\}=\min \left\{-\mu[T P(\mathbf{x}, \mathbf{u})], \sigma^{2}[T P(\mathbf{x}, \mathbf{u})]\right\} \\
& \mathbf{x}=\left[x_{1}, x_{2}, x_{3}\right], \mathbf{u}=\left[u_{1}, u_{2}\right] \\
& \mu(\mathbf{x}, \mathbf{u}) \geq \mu(\text { Ref }) \\
& \sigma^{2}(\mathbf{x}, \mathbf{u}) \leq \sigma^{2}(\text { Ref })
\end{aligned}
$$


For $\mathrm{RO}$ of $\mathrm{VG}$, the population size and the generations of SMODE algorithm are set as 100 and 300, respectively. Figures 11 show the Pareto front of basic RO, both the mean and variance of the optimal Pareto solutions are far better than those of the reference design, it implies that the constraints based on reference design can be easily met.

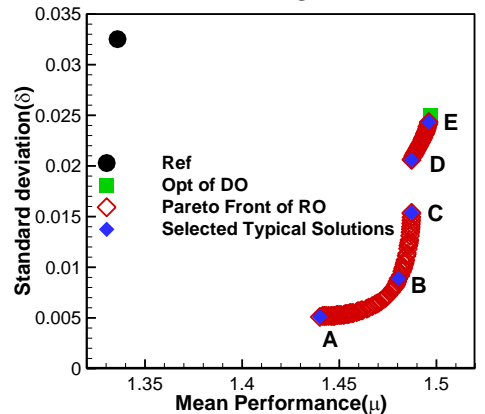

Fig.11 Pareto front of basic RO process

In Fig.12, the parallel axes (PA) was used to visualize the variable space of optimal solutions [21]. The PA has one horizontal axis and several vertical axes. The points in each vertical axis show the value distributions of each component variable $\left(x_{1}, x_{2}, x_{3}\right)$ and the performance indicators $\left(\mu, \sigma^{2}\right)$ as well, the polyline that connects the points show the value distribution of each solution. Further, the polylines are colored according to the the mean value $(\mu)$ of solutions to shows the combined effects of variables more clearly.

As shown in Fig. 12, the solutions of optimal Pareto front are basically divided into two groups, with very different distributions of $W$ and $H$. This explains why the Pareto fronts in Fig. 11 are distributed in two clusters. In other words, the variable space of optimal solutions is multi-island, similar to the multi-modal problem in single-objective optimization, in order to fetch all potential good solutions, it requires the optimization algorithms has excellent global search performance .

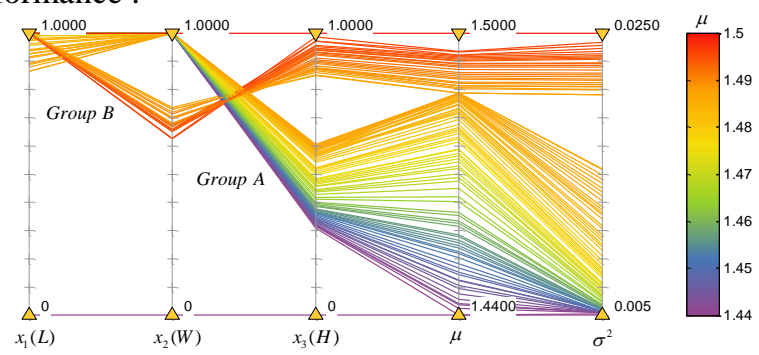

Fig. 12 Variable space of optimal Pareto solutions of basic RO

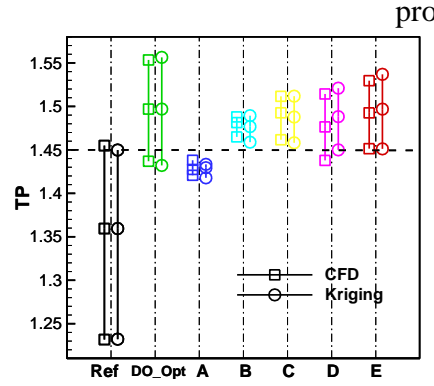

(a) Validation for kriging with adaptive sampling

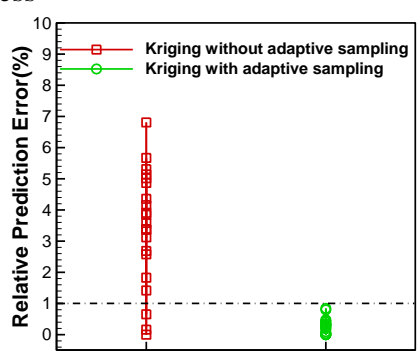

(b) Kriging with/without adaptive sampling
Fig.13 CFD Verification of the best, worst and nominal performance of typical designs
Further, several typical optimal solutions as Designs A E in Fig.11 are selected to validate their performance w.r.t. those of the reference design. Obviously, the prediction of the kriging with adaptive sampling (using Eq. (10)) agrees well with CFD results, as shown in Fig.13 (a), the largest relative prediction error is $0.84 \%$. On the other hand, the accuracy of kriging without adaptive sampling was shown much worse in Fig.13 (b). It confirms that the adaptive sampling is effective to improve the surrogate accuracy.

Then, the solutions of DO and RO are compared through Figs. 11 and 13. As shown in Fig. 11, the DO_Opt has largest variance, though its mean performance is also the best. From Fig.13, we further observe that the TP of DO_Opt fluctuates in a very large range as $[1.4369,1.5534]$, the best TP of DO_Opt is increased by $8.47 \%$ w.r.t. its worst TP, while the TP increment of $5 \%$ should be considerable in design practice [29, 30]. It means that, in order to find solutions that are not so sensitive to uncertainty fluctuates, the RO is demanded.

Regarding the design robustness, Designs $\mathrm{A}$ and $\mathrm{B}$ in Fig.13 should be the desirable RO solutions, as their performance fluctuations are small. However, when comparing the TP of Design A and DO_Opt at different uncertainty conditions, the worst TP of DO_Opt is even better than the best TP of Design A, i.e., the TP Design A is inferior to DO_Opt over the uncertainty fluctuation range. Then, the designers may hesitate, whether or not to use instead of DO_Opt? As a contrast, the performance of Design $\mathrm{B}$ is much more competitive, in addition to robustness.

Then, the question arise, could we automatically filter out “inferior" RO solutions like Design A? The constraints set based on information of DO_Opt may do the help, which will be discussed in detail in the next subsection.

\section{RO Strategies by Considering Solution of DO}

Before the discussions on adding constraints based on information of DO_Opt, we first discuss which kind of RO solutions should be most favorable.

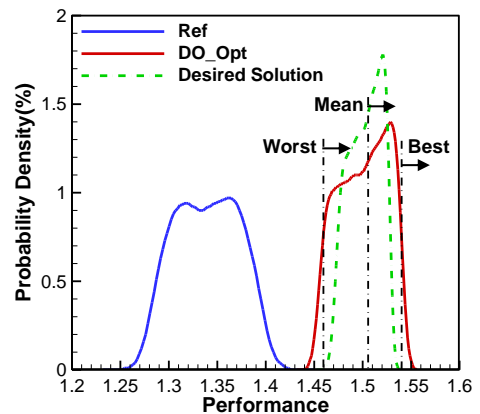

Fig.14 Desirable solutions of RO in kernel density plot

Figure 14 shows the kernel density plot of the reference design and DO_Opt, it presents the possibilities (or frequencies) of each TP appeared in a solution when the uncertainties propagates. Considering design robustness to uncertainties that come from manufacturing error or environmental variation, the solution curve with narrower width with higher peaks is most desirable. In addition, to have competitive TP, we should also impose constraints on 
the worst, mean and best TP of the solution candidates, i.e. the mean, worst and best TP of the solution canidate should be better than a certain value, and the larger is better. Then, Table 6 lists the information of DO_Opt and the reference design, where Q1 and Q3 are the first and the third quartile of the performance datasets, these values of DO_Opt are used as constraints to inspire better solutions.

Table 6 Statistical information of reference design and DO_Opt

\begin{tabular}{ccccccc}
\hline & $\mu$ & $\sigma$ & Min & Max & Q1 & Q3 \\
\hline Ref & 1.3359 & 0.0325 & 1.2521 & 1.4299 & 1.3127 & 1.3661 \\
DO_Opt & 1.4968 & 0.0250 & 1.4369 & 1.5534 & 1.4797 & 1.5231 \\
\hline
\end{tabular}

By taking the information of DO_Opt as constraints, Table 7 lists nine RO strategies; however, not all of them can obtain solutions, i.e., the solution set can be null as shown in the last column. To be noted, the RO space is a black-box, few prior knowledge can be obtained on whether the imposed constraints is too strong, therefore out of the nine RO models in Table.7, null solution sets were encountered three times (e.g. Strategy 2a,4a and 5a) It means, the RO would be an iteration process, we cannot guarantee the proposed RO model can always found solutions, i.e., we may have to conduct RO process several times, therefore it is attractive to build high-accurate surrogate before RO, which can efficiently handle RO models with different constraints.

Table $7 \quad$ RO strategies by considering solution of DO

\begin{tabular}{|c|c|c|c|}
\hline Strategies & No. & Fomulations of RO models & Results \\
\hline $\begin{array}{l}\text { Constraints on } \\
\text { the mean } \\
\text { design } \\
\text { performance }\end{array}$ & 1 & $\begin{array}{c}\min \left\{-\mu(\mathbf{x}, \mathbf{u}), \sigma^{2}(\mathbf{x}, \mathbf{u})\right\} \\
\text { s.t. } \mu(\mathbf{x}, \mathbf{u}) \geq Q 1\left(D O_{-} O p t\right)\end{array}$ & Fig.17(a) \\
\hline \multirow{2}{*}{$\begin{array}{l}\text { Constraints on } \\
\text { the worst } \\
\text { performance }\end{array}$} & $2 \mathrm{a}$ & $\begin{array}{c}\min \left\{-\mu(\mathbf{x}, \mathbf{u}), \sigma^{2}(\mathbf{x}, \mathbf{u})\right\} \\
\text { s.t. } f_{\min }(\mathbf{x}, \mathbf{u}) \geq Q 1\left(D O_{-} O p t\right)\end{array}$ & Null \\
\hline & $2 b$ & $\begin{array}{c}\min \left\{-\mu(\mathbf{x}, \mathbf{u}), \sigma^{2}(\mathbf{x}, \mathbf{u})\right\} \\
\text { s.t. } f_{\min }(\mathbf{x}, \mathbf{u}) \geq \min \left(D O_{-} O p t\right)\end{array}$ & Fig.17(b) \\
\hline $\begin{array}{l}\text { Constraints on } \\
\text { the best } \\
\text { performance }\end{array}$ & 3 & $\begin{array}{l}\min \left\{-\mu(f(\mathbf{x}, \mathbf{u})), \sigma^{2}(f(\mathbf{x}, \mathbf{u}))\right\} \\
\text { s.t. } f_{\max }(\mathbf{x}, \mathbf{u}) \geq Q 3\left(D O_{-} O p t\right)\end{array}$ & Fig.17(c) \\
\hline \multirow{2}{*}{$\begin{array}{l}\text { Constraints on } \\
\text { both the mean } \\
\text { and the worst } \\
\text { performance }\end{array}$} & $4 a$ & $\begin{array}{c}\min \left\{-\mu(\mathbf{x}, \mathbf{u}), \sigma^{2}(\mathbf{x}, \mathbf{u})\right\} \\
\text { s.t. } f_{\min } \geq \min \left(D O_{-} O p t\right) \\
\mu(\mathbf{x}, \mathbf{u}) \geq \mu\left(D O_{-} O p t\right)\end{array}$ & Null \\
\hline & $4 b$ & $\begin{array}{ll} & \min \left\{-\mu(\mathbf{x}, \mathbf{u}), \sigma^{2}(\mathbf{x}, \mathbf{u})\right\} \\
\text { s.t. } & f_{\min }(\mathbf{x}, \mathbf{u}) \geq \min \left(D O_{-} O p t\right) \\
& \mu(\mathbf{x}, \mathbf{u}) \geq Q 1\left(D O_{-} O p t\right)\end{array}$ & Fig.17(d) \\
\hline \multirow{2}{*}{$\begin{array}{l}\text { Constraints on } \\
\text { both the best } \\
\text { and the worst } \\
\text { performance }\end{array}$} & $5 \mathrm{a}$ & $\begin{array}{c}\min \left\{-\mu(\mathbf{x}, \mathbf{u}), \sigma^{2}(\mathbf{x}, \mathbf{u})\right\} \\
\text { s.t. } \\
f_{\min }(\mathbf{x}, \mathbf{u}) \geq \min \left(D O_{-} O p t\right) \\
f_{\max }(\mathbf{x}, \mathbf{u}) \geq Q 3\left(D O_{-} O p t\right)\end{array}$ & Null \\
\hline & $5 b$ & $\begin{array}{c}\min \left\{-\mu(\mathbf{x}, \mathbf{u}), \sigma^{2}(\mathbf{x}, \mathbf{u})\right\} \\
\text { s.t. } \\
f_{\min }(\mathbf{x}, \mathbf{u}) \geq \min \left(D O_{-} O p t\right) \\
f_{\max }(\mathbf{x}, \mathbf{u}) \geq \mu\left(D O_{-} O p t\right)\end{array}$ & Fig.17(d) \\
\hline $\begin{array}{l}\text { Constraints on } \\
\text { the mean, best } \\
\text { and worst } \\
\text { performance }\end{array}$ & 6 & $\begin{array}{c}\min \left\{-\mu(\mathbf{x}, \mathbf{u}), \sigma^{2}(\mathbf{x}, \mathbf{u})\right\} \\
\text { s.t. } f_{\min }(\mathbf{x}, \mathbf{u}) \geq \min \left(D O_{-} O p t\right) \\
f_{\max }(\mathbf{x}, \mathbf{u}) \geq \mu\left(D O_{-} O p t\right) \\
\mu(\mathbf{x}, \mathbf{u}) \geq Q 1\left(D O_{-} O p t\right)\end{array}$ & Fig.17(d) \\
\hline
\end{tabular}

For the nine RO strategies shown in Table 7, four different Pareto solutions were finally found as shown in Fig.15. Where, the optimal solutions in Fig.15 (a) (Strategy 1) were obtained by setting the threshold that the mean value of candidate RO solutions should be better than Q1 of DO_Opt (see Table 6). The optimal solutions in Fig.15 (b) (Strategy $2 \mathrm{~b}$ ) were achieved by requiring that the worst performance of candidate RO solutions should be better than the worst performance of DO_Opt. The optimal solutions in Fig.15(c) (Strategy 2c) were selected by filtering out the solutions whose best performance worse than Q3 of DO_Opt. And finally, the solutions in Fig.15 (d) can be obtained by the strategies 4b, 5b and 6; they impose requirements on the both the worst and best and even the mean performance of candidate RO solutions.

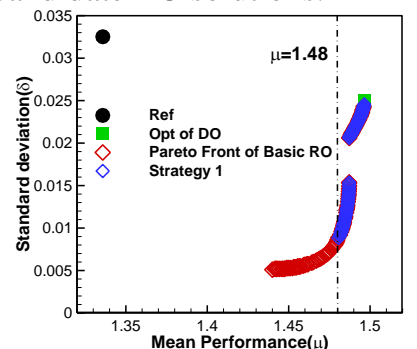

(a) Strategy 1

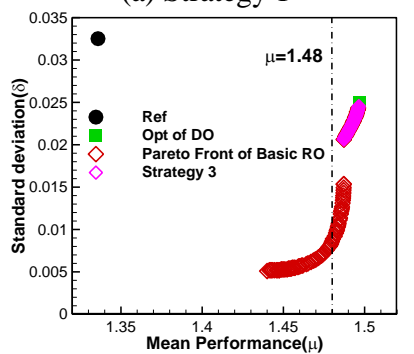

(c)Strategy 3

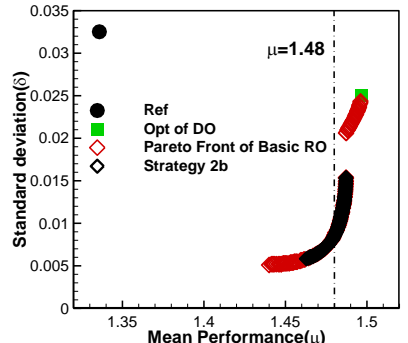

(b) Strategy $2 b$

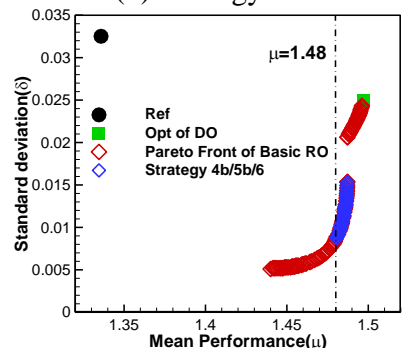

(d) Strategy $4 \mathrm{~b} / 5 \mathrm{~b} / 6$
Fig.15 Optimal Pareto solutions of different RO strategies

In addition to finding desirable $\mathrm{RO}$ solutions, the solutions of RO models with different constraints also provide a way to get an insight into the variable space of optimal solutions, which are illustrated by combining Figs. 15 to 17. The optimal solutions of basic RO can actually be divided into four clusters as shown in Fig.16, and Fig.17 presents the performance of typical solutions in each cluster. The solutions in Cluster IV are obtained by Strategy 3, their features can be observed by comparing Fig.15 (a), (c) and (d), these solutions have good mean performance but large performance fluctuations as well, their worst performance is even worse than the worst performance of DO_Opt, as seen by the typical solution IVD in Fig. 17.

The solutions in Cluster III are obtained by Strategy $4 \mathrm{~b}$, they should be most desirable our problem in basic RO space. As shown by the solution IIIC in Fig.17, their mean and worst performance are competitive to those of DO_Opt, and the performance fluctuations are also small. The solutions in Cluster II can be competitive to those in Cluster III, their best performance is inferior to those in Cluster III (comparing Strategy $2 b$ and $4 b$ ), however, their performance are less sensitive to the uncertainties propagates, as shown by solution IIB. 
The solutions in Cluster I may be not so competitive, though their performance is most insensitive to the uncertainties, the performance values are much lower, the best TP can be even worse than the worst of DO_Opt.

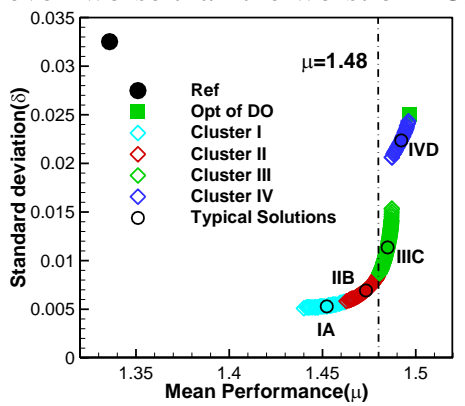

Fig.16 Clustering of the Pareto Solutions in basic RO space

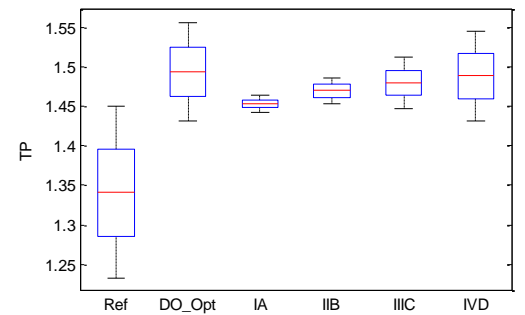

Fig. 17 Boxplot of performance fluctuations of typical designs in each clusters with uncertainty variations

Finally, by referring to the variable space shown by parallel axis in Fig.12, we found the solutions in Cluster IV belongs to Group B, characterized by larger mean and variance values. The solutions in Clusters I, II and III belongs to Group A, of which performance are less sensitive to the uncertainty fluctuates but have worse TP values. Regarding to geometric features of $\mathrm{VG}$, the desirable solutions in Cluster III should have large $L$ and $W$, while the $H$ seems crucial to determine the overall thermal performance of VG, as seen in Fig.12. For the VGs in Group $\mathrm{B}$ (Cluster IV), the $\mathrm{W}$ also plays an important role in addition to $H$. More technical investigations on the flow mechanism behind the geometric features of VGs will be discussed in our future work.

\section{CONCLUSION}

By combining a kriging-based uncertainty quantification (UQ) formulation for the mean and variance and a selfadaptive multi-objective optimization (SMODE) algorithm, a multi-objective robust optimization (RO) platform was proposed, which aimed for the situation when uncertainty parameters and optimization design variables cannot be merged as uncertainty variable. The RO platform was used for the optimization of vortex generators (VG)) to enhance the overall heat transfer performance of U-bend channel for high-temperature blades, with one Gaussian-distributed and one uniformly-distributed uncertainty parameters, and three optimization design variables. Several conclusions were made as follows:

The kriging-based mean-variance UQ formulation formulates the uncertainty parameters and optimization design variables in product form, and thus optimization search and UQ are conducted simultaneously in RO. The product form makes the UQ formula can assign different probability distribution functions to each uncertainty parameter; it also makes the integral dimension to be low and thus can be efficiently calculated.

For the RO platform, a high-accurate surrogate is built before RO. The sequential sampling strategies, which originally used in efficient optimization, were employed to improve the surrogate accuracy, which was shown effective in the five-variable VG problem. Since the RO process may have to iterate several times as shown in the VG design, it is attractive to build high-accurate surrogate before RO.

For the robust design of $\mathrm{VG}$, one deterministic optimization (DO) and several RO were conducted. When compared to DO, the RO was shown able to find robust solutions, which have high performance and is not sensitive to the uncertainty fluctuations. Meanwhile, the uncertainty information of the solution of DO provides good reference to exclude insensitive solutions which actually have much lower design performance. Alternatively, we may use other performance thresholds as required by engineering products to select solutions with favorite design performance.

The RO can be an iterative process for black-box problems, as some strong constraints may be imposed to make the solution set to be null. From another perspective, the solutions of RO models with different constraints also provide a way to get insights into the black-box design space, which is helpful to select the final solution for engineering use.

Finally, from the perspective of enhancing the heat transfer performance in U-bend channel of high-temperature blades, the optimized VG was shown have better overall thermal performance than classic cooling structures such as angled ribs. We summarized the geometric features of desirable VGs by parallel axis, the related flow mechanism behind will be analyzed in our future work.

\section{REFERENCES}

[1] Park, G. J., Lee, T. H., Lee, K. H., \& Hwang, K. H., "Robust Design: an Overview," AIAA Journal, Vol.44, No.1, 2006, pp.181191.

[2] Bunker R S., "The effects of manufacturing tolerances on gas turbine cooling," ASME Journal of Turbomachinery, Vol.131, No.4, 2009, pp. 041018.

[3] D'Ammaro A. Montomoli F., "Uncertainty quantification and film cooling," Computers \& Fluids, Vol.71, 2011, pp. 320-326.

[4] Beyer, H. G., \& Sendhoff, B., "Robust optimization-a comprehensive survey," Computer Methods in Applied Mechanics and Engineering, Vol.196, No.33-34, 2007, pp.3190-3218.

[5] Yang J, Xiong J, McBean I, Havakechian S, Liu F, and Luo J. Performance impact of manufacturing variations for multi-stage steam turbines. AIAA Journal of Propulsion and Power, 2017, Vol.33, No.4, 2017, pp.1031-1036.

[6] Kim NK, Kim DH, Kim DW, Kim HG, Lowther D, Sykulski JK., "Robust optimization utilizing the second-order design sensitivity information," IEEE Transitions on, Vol. 46,No.8,2010, pp.3117-3120.

[7] Lange, A., Voigt, M., Vogeler, K., Schrapp, H., Johann, E., \& Gümmer, V., "Impact of manufacturing variability and 
nonaxisymmetry on high-pressure compressor stage performance," ASME Journal of Engineering for Gas Turbines and Power, Vol.134, No.3, 2012, pp. 032504.

[8] Palar PS, Shimoyama K, "Multi-fidelity Uncertainty Analysis in CFD using Hierarchical Kriging,"AIAA AVIATION Forum, 35th AIAA Applied Aerodynamic Conference, June 5-9, 2017, Denver, Colorado, U.S.A.

[9] Huang T, Song X, Liu X, "The Multi-objective Robust Optimization of the Loading Path in the T-shape Tube Hydroforming Based on Dual Response Surface Model, " International Journal of Advance Manufacturing Technology, Vol.82, No.9-12, 2015, pp.1-11.

[10] Liu, Y, Bai JQ and Livne E., "Robust Optimization of Variable-Camber Continuous Trailing-Edge Flap Static Aeroelastic Action," AIAA Journal, Vol.55, No. 3, 2017, pp. 1031-1043.

[11] Kim NH, Wang H, Queipo NV, "Efficient Shape Optimization under Uncertainty Using Polynomial Chaos Expansions and Local Sensitivities," AIAA Journal, Vol. 44, No. 5, 2006, pp. 1112-1116.

[12] Padron AS, Alonso JJ, Eldred MS, "Multi-fidelity Methods in Aerodynamic Robust Optimization," AIAA SciTech Forum, 18th AIAA Non-Deterministic Approaches Conference, January, 4-8, 2016, San Diego, California, USA.

[13] Arendt PD, Apley DW, Chen W, "Objective-Oriented Sequential Sampling for Simulation Based Robust Design Considering Multiple Sources of Uncertainty ," ASME Journal of Mechanical Design, Vol.135, No.5,2013, pp. 051005-1-10

[14] Zhou Q., Wang Y., Choi SK, Jiang P., Shao X., Hu J. and Shu L. "A Robust Optimization Approach Based on Multi-fidelity Metamodel," Structural Multi-disciplinary Optimization, Vol.57, No.2, 2018, pp.775-797.

[15] Sun G, Song X, Baek S, Li Q. "Robust Optimization of Foamfilled Thin-walled Structure Based on Sequential Kriging metamodel," Structural Multidisciplinary Optimization, Vol.49, No.6, pp.897-913.

[16] Lian Y and Kim N.H., "Reliability-Based Design Optimization of a Transonic Compressor," AIAA Journal, Vol. 44, No. 2, 2006, pp. 368-375.

[17] Keane, A. J., "Comparison of several optimization strategies for robust turbine blade design," AIAA Journal of Propulsion and Power, Vol.25, No.5, 2009, pp. 1092-1099.

[18] Ryan, K. M., Lewis, M. J., \& Yu, K. H, "Comparison of robust optimization methods applied to hypersonic vehicle design," AIAA Journal of Aircraft, Vol.52, No.5, 2015, pp.1510-1523.

[19] Shan S.Q. and Wang G.G. ,"Survey of modeling and optimization strategies to solve high-dimensional design problems with computationally-expensive black-box functions." Structural and Multidisciplinary Optimization, Vol. 41, No. 2 2010, pp. 219 241.

[20] Guo Z., Song L, Zhou Z, Li J, Feng Z., "Multi-objective aerodynamic optimization design and data mining of a high pressure ratio centrifugal impeller," ASME Journal of Engineering for Gas Turbines and Power, 2015, 137(9): 092602-1-14.

[21] Song L., Guo Z, Li J and Feng Z," Research on MetamodelBased Global Design Optimization and Data Mining Methods," ASME Journal of Engineering for Gas Turbines and Power, 2016, Vol.138, No. 9, pp.092604-1-14.
[22] Schonlau M. "Computer experiments and global optimization," Doctor Dissertation, University of Waterloo, 1997.

[23] Viana, F.A.C, Haftka, R.T., and Watson, L.T., "Efficient global optimization algorithm assisted by multiple surrogate techniques" Journal of Global Optimization, 2013, Vol. 56, pp. 669689.

[24] Zhan D., Qian J. Cheng Y.S., "Balancing global and local search in parallel efficient global optimization algorithms," Journal of Global Optimization, 2017, Vol.57, No.4, pp. 873-892.

[25] Surjanovic, S. \& Bingham, D. (2013). Virtual Library of Simulation Experiments: Test Functions and Datasets. Retrieved August 1, 2018, from http://www.sfu.ca/ ssurjano.

[26] Schonlau M. and Welch WJ," Screening the Input Variables to a Computer Model via Analysis of Variance and Visualization Screening,” 2006, Springer, New York, pp.308-327.

[27] Ponweiser W, Wagner T, Vincze M." Clustered Multiple Generalized Expected Improvement: A novel Infill Sampling Criterion for Surrogate Models," IEEE World Congress on Computational Intelligence, Hong Kong, China, 2008: pp.35153522 .

[28] Song L,M. Luo C, Li J, Feng Z.P., “Automated Multiobjective and Multidisciplinary Design Optimization of a Transonic Turbine Stages," Journal of Power and Energy, 2012, Vol.226, No.2, pp. 262-276.

[29] Liu Y H, Wright L M, Fu W L, Han J.C., "Rib Spacing Effect on Heat Transfer and Pressure Loss in a Rotating Two-Pass Rectangular Channel (AR= 1: 2) with 45-Degree Angled Ribs," ASME Turbo Expo: Power for Land, Sea, and Air. American Society of Mechanical Engineers, 2006, May 8-11, 2006, Barcelona, Spain, ASME Paper GT2006-90368, 2006.

[30] Henze M, Dietz C, von Wolfersdorf J, Bernhard W., "Heat Transfer around Longitudinal and Parallel Arranged Wedge-shaped Vortex Generators," ASME Turbo Expo 2007: Power for Land, Sea, and Air. American Society of Mechanical Engineers, 2007, pp. 693702.

[31] ANSYS, 2010, ANSYS CFX-Solver Theory Guide, Release 13.0.ANSYS Inc., Canonsburg, PA 